PROCEEDINGS OF THE AMERICAN MATHEMATICAL SOCIETY

Volume 126, Number 3, March 1998, Pages 629-635

S $0002-9939(98) 04521-3$

\title{
TWO FINITELY GENERATED VARIETIES HAVING NO INFINITE SIMPLE MEMBERS
}

\author{
ROSS WILLARD
}

(Communicated by Lance W. Small)

\begin{abstract}
Using a method of R. McKenzie, we construct a finitely generated semisimple variety of infinite type, and a finitely generated nonsemisimple variety of finite type, both having arbitrarily large finite but no infinite simple members. This amplifies M. Valeriote's negative solution to Problem 11 from Hobby and McKenzie, The Structure of Finite Algebras.
\end{abstract}

R. McKenzie [2] has constructed a finitely generated variety having arbitrarily large finite, but no infinite, subdirectly irreducible members; and a second finitely generated variety, this time of finite type, having a unique countably infinite subdirectly irreducible member but no uncountable subdirectly irreducible members. M. Valeriote [4] has subsequently shown that McKenzie's second example can be modified to make the variety semisimple, i.e., so that every subdirectly irreducible member is simple. (In Valeriote's example there are three, rather than one, countably infinite simple members.)

In this note we (1) show that McKenzie's first example can also be modified to make the variety semisimple; (2) modify McKenzie's second example in a different way to obtain a finitely generated (nonsemisimple) variety of finite type having arbitrarily large finite, but no infinite, simple members.

\section{MCKenZiE's Method}

The following is a summary of a method invented by McKenzie in $[2, \S 6]$, as it is described in [5]. An $\mathbf{M}$-algebra is any algebra $\mathbf{A}$ whose type includes $\wedge$ (binary) and 0 (nullary) but no other nullary operation symbols, and which satisfies

1. The reduct $\langle A, \wedge\rangle$ is a height- 1 meet semilattice with least element 0 ;

2. 0 is an absorbing element for each fundamental operation $F$ of $\mathbf{A}$; that is, if $F$ is $n$-ary then $0 \in\left\{a_{1}, . ., a_{n}\right\} \subseteq A$ implies $F\left(a_{1}, \ldots, a_{n}\right)=0$.

Suppose $\mathbf{A}$ is an M-algebra and $U=A \backslash\{0\}$. Consider an arbitrary subpower $\mathbf{B} \leq \mathbf{A}^{I}(I \neq \emptyset)$ with $B(U):=B \cap U^{I} \neq \emptyset$. On $B(U)$ define the (reflexive) binary relation $\gg$ by $f \gg g$ if and only if $F^{\mathbf{B}}\left(h_{1}, \ldots, h_{n}\right)=g$ for some fundamental operation symbol $F$ and some $h_{i} \in B(U)$ such that $f \in\left\{h_{1}, \ldots, h_{n}\right\}$. Also let

Received by the editors October 26, 1995.

1991 Mathematics Subject Classification. Primary 08B26.

Key words and phrases. Variety, finitely generated, simple, semisimple.

The support of the NSERC of Canada is gratefully acknowledged.

(C)1998 American Mathematical Society 
be the transitive closure of $\gg$. Now choose any $p \in B(U)$ and let

$$
B_{p}=\{f \in B(U): f \ggg p\} .
$$

$\mathbf{B}(p)$ denotes the M-algebra, of the same type as $\mathbf{A}$ and $\mathbf{B}$, whose universe is the disjoint union of $B_{p}$ and $\{0\}$, and whose fundamental operations are defined as follows:

$$
F^{\mathbf{B}(p)}\left(h_{1}, \ldots, h_{n}\right)= \begin{cases}F^{\mathbf{B}}\left(h_{1}, \ldots, h_{n}\right) & \text { if } F^{\mathbf{B}}\left(h_{1}, \ldots, h_{n}\right) \in B_{p}, \\ 0 & \text { otherwise }\end{cases}
$$

$\mathbf{B}(p)$ is subdirectly irreducible, and is simple if and only if the restriction of $\ggg$ to $B_{p}$ is symmetric.

Next, we describe two kinds of "conditions" which can be imposed on $\mathbf{B}(p)$. Suppose that $\bar{x}$ is a finite sequence of distinct variables, that $\Sigma(\bar{x})$ is a conjunction of equations $x_{i}=x_{j}$ between pairs of these variables, that $s(\bar{x}), t(\bar{x})$ are terms in these variables, and that each variable in $\bar{x}$ occurs explicitly in $s$ or $t$. The " $\mathcal{C}$ condition corresponding to $s, t, \Sigma$ " is the following first-order sentence, denoted by $\mathcal{C}_{\Sigma}^{s=t}$ :

$$
(\forall \bar{x})(s(\bar{x})=t(\bar{x}) \neq 0 \Rightarrow \Sigma(\bar{x})) .
$$

We say that $(\mathbf{B}, p)$ satisfies $\mathcal{C}_{\Sigma}^{s=t}$ if this sentence is true in $\mathbf{B}(p)$.

Secondly, suppose $\Phi(\bar{x})$ is an $n$-ary predicate on $U$. The " $\mathcal{B}$-condition corresponding to $\Phi$, , denoted by $\mathcal{B}_{\Phi}$, is the following condition on $\mathbf{B}$ :

There do not exist $f_{1}, \ldots, f_{n} \in B(U)$ such that $\Phi\left(f_{1}(i), \ldots, f_{n}(i)\right)$ is true at every coordinate $i \in I$.

We say that $(\mathbf{B}, p)$ satisfies $\mathcal{B}_{\Phi}$ when the above condition holds.

Theorem 1.1 (McKenzie's Method, weak version; see [5, Theorem 2.1]). Suppose A is a finite $M$-algebra with $U=A \backslash\{0\}$, and that $\mathcal{X}$ is a collection of $\mathcal{B}$ - and $\mathcal{C}$ conditions. Let $\mathcal{K}_{1}$ be the class of all algebras $\mathbf{B}(p)$ where $\mathbf{B} \leq \mathbf{A}^{I}, p \in B(U)$, and $(\mathbf{B}, p)$ satisfies all of the conditions in $\mathcal{X}$, and let $\mathcal{K}_{2}=\left\{\mathbf{B}(p) \in \mathcal{K}_{1}: B_{p}=B(U)\right\}$. Finally, let $\mathcal{U}$ be the class of all algebras of the same type as $\mathbf{A}$.

There exist (i) a type $\tau$, (ii) a variety $\mathcal{W}$ of type $\tau$, (iii) a term equivalence (in the sense of [3, Def. 4.138]) from $\mathcal{U}$ to $\mathcal{W}$, which we shall denote in the forward direction by $\mathbf{D} \mapsto \mathbf{D}^{\otimes}$, and (iv) a finite algebra $\mathbf{A}_{1}^{+}$of type $\tau$, which satisfy:

1. $\mathcal{K}_{2}^{\otimes}:=\left\{\mathbf{B}(p)^{\otimes}: \mathbf{B}(p) \in \mathcal{K}_{2}\right\}$ is contained in $\mathbf{V}\left(\mathbf{A}_{1}^{+}\right)$;

2. Conversely, every subdirectly irreducible member of $\mathrm{V}\left(\mathbf{A}_{1}^{+}\right) \backslash \mathrm{HS}\left(\mathbf{A}_{1}^{+}\right)$is isomorphic to a member of $\mathcal{K}_{1}^{\otimes}$;

3. $\tau$ is finite if and only if both $\mathcal{X}$ and the type of $\mathbf{A}$ are finite.

\section{Semisimple VARIETy OF INFINITE TYPE}

Let $U_{0}=\{a, b\}$ and, for each $n \geq 3$, let $\Phi_{n}\left(x_{0}, \ldots, x_{n}\right)$ be the $(n+1)$-ary predicate on $U_{0}$ defined by

For $x_{0}, \ldots, x_{n} \in U_{0}$, let $T=\left\{i \leq n: x_{i}=b\right\}$. Then $\Phi_{n}(\bar{x})$ if and only if either $x_{0}=a$ and $|T|=1$ or $n$, or $x_{0}=b$ and $T$ is an initial segment of $\{0,1, \ldots, n\}$. 
McKenzie showed [2, Theorem 1.1] that for each $n \geq 3$ there is a set $I$ and an $(n+1)$-element subset $R_{n}=\left\{\beta_{0}, \beta_{1}, \ldots, \beta_{n}\right\} \subseteq U_{0}^{I}$ with the following properties:
(*) 2. For all $m \geq 3$ with $m \neq n$, there do not exist $f_{0}, \ldots, f_{m} \in R_{n}$ satisfying $\Phi_{m}(\bar{f})$.
3. $\hat{a} \notin R_{n}$. ( $\hat{a}$ is the constant function in $U_{0}^{I}$ with value $a$.)

Let $U=\{1, a, b\}, A=\{0,1, a, b\}$, and for each $n \geq 3$ define $F_{n}: A^{n+1} \rightarrow A$ by

$$
F_{n}(\bar{x})= \begin{cases}1 & \text { if } \Phi_{n}(\bar{x}) \\ 0 & \text { otherwise }\end{cases}
$$

Also for each $n \geq 3$ and $i \leq n$ define $G_{n, i}: A^{n+2} \rightarrow A$ by

$$
G_{n, i}(\bar{x}, y)=\left\{\begin{array}{cl}
x_{i} & \text { if } \Phi_{n}(\bar{x}) \text { and } y=1 \\
0 & \text { otherwise. }
\end{array}\right.
$$

Finally, let $P, Q$ be the functions $A^{2} \rightarrow A$ defined by $P(1, a)=1, Q(1, a)=a$, and $P(x, y)=Q(x, y)=0$ in all other cases. Define $\mathbf{A}$ to be the M-algebra with universe $A$ and operations $\wedge, 0, P, Q, F_{n}(n \geq 3)$, and $G_{n, i}(n \geq 3, i \leq n)$.

For $3 \leq n<m<\omega$ define

$$
\begin{aligned}
\mathcal{C}_{n} & =\mathcal{C}_{\& x_{i}=y_{i}}^{\mathrm{F}_{n}(\bar{x})=\mathrm{F}_{n}(\bar{y})} \\
\mathcal{B}_{n, m} & =\mathcal{B}_{\Phi_{n}(\bar{x}) \& \Phi_{m}(\bar{y})},
\end{aligned}
$$

and put

$$
\mathcal{X}=\left\{\mathcal{B}_{n, m}: 3 \leq n<m<\omega\right\} \cup\left\{\mathcal{B}_{x=a}\right\} \cup\left\{\mathcal{C}_{n}: 3 \leq n<\omega\right\} .
$$

Lemma 2.1. Suppose that $\mathbf{B} \leq \mathbf{A}^{I}, p \in B(U)$, and $\left|B_{p}\right|>1$. Then

1. $\hat{1} \in B_{p}$.

2. $(\mathbf{B}, p)$ satisfies the conditions in $\mathcal{X}$ if and only if (i) there is a solution $\bar{f}$ to $\Phi_{n}(\bar{x})$ in $B(U)$ for exactly one value of $n$; (ii) for that $n$, there is exactly one solution $\bar{f}$ to $\Phi_{n}(\bar{x})$ in $B(U)$; (iii) $\hat{a} \notin B(U)$.

Proof. Since $\left|B_{p}\right|>1$ there exists $h \in B(U)$ such that $h \neq p$ and $h \gg p$. Thus either $P\left(g, g^{\prime}\right)=p$ or $Q\left(g, g^{\prime}\right)=p$ for some $g, g^{\prime} \in B(U)$, or $F_{n}(\bar{f})=p$ or $G_{n, i}(\bar{f}, g)=p$ for some $n \geq 3, i \leq n$, and $f_{0}, \ldots, f_{n}, g \in B(U)$. In the first and second cases we must have $g^{\prime}=\hat{a}$ and $\hat{1}=g \gg p$. In the third case we must have $\Phi_{n}(\bar{f})$ and $\hat{1}=p$, while in the last case we must have $\Phi_{n}(\bar{f})$ and $\hat{1}=g \gg p$. Thus $\hat{1} \gg p$ in every case, which proves the first item.

$(\mathbf{B}, p)$ satisfies $\mathcal{B}_{x=a}$ if and only if $\hat{a} \notin B(U)$. Assume $\hat{a} \notin B(U)$. Then the first two cases in the previous paragraph cannot hold. Thus there must exist an $n \geq 3$ and a solution $\bar{f}$ to $\Phi_{n}(\bar{x})$ in $B(U)$. The remaining $\mathcal{B}$-conditions in $\mathcal{X}$ are equivalent in $(\mathbf{B}, p)$ to the requirement that for every $m \neq n, \Phi_{m}(\bar{x})$ have no solutions in $B(U)$.

Assume now that $(\mathbf{B}, p)$ satisfies all of the $\mathcal{B}$-conditions. Then $(\mathbf{B}, p)$ also satisfies $\mathcal{C}_{m}$ for every $m \neq n$, since $\mathbf{B}(p) \models F_{m}(\bar{g}) \neq \hat{0}$ only if $\Phi_{m}(\bar{g})$. Finally, note that all solutions to $\Phi_{n}(\bar{x})$ in $B(U)$ must belong to $B_{p}$. This is so because $\Phi_{n}(\bar{g})$ implies $F_{n}(\bar{g})=\hat{1}$ and hence $g_{0}, \ldots, g_{n} \gg \hat{1} \in B_{p}$. Thus $(\mathbf{B}, p)$ will satisfy $\mathcal{C}_{n}$ if and only if $\bar{f}$ is the unique solution to $\Phi_{n}(\bar{x})$ in $B(U)$.

Let $\mathcal{K}_{1}$ be the class of all algebras $\mathbf{B}(p)$ where $\mathbf{B} \leq \mathbf{A}^{I}(I \neq \emptyset), p \in B(U)$, and $(\mathbf{B}, p)$ satisfies the conditions in $\mathcal{X}$, and let $\mathcal{K}_{2}=\left\{\mathbf{B}(p) \in \mathcal{K}_{1}: B_{p}=B(U)\right\}$. 
Lemma 2.2. For every $k \geq 6$ there exists a $k$-element member of $\mathcal{K}_{2}$.

Proof. Let $n=k-3$ and choose $I \neq \emptyset$ and $R_{n}=\left\{\beta_{0}, \ldots, \beta_{n}\right\} \subseteq U_{0}^{I}$ witnessing $(*)$. Let $B=\left(A^{I} \backslash U^{I}\right) \cup R_{n} \cup\{\hat{1}\}$ and $p=\hat{1}$. Then $\mathbf{B}$ is a subalgebra of $\mathbf{A}^{I}$, $B_{p}=B(U)=R_{n} \cup\{\hat{1}\}$, and $(\mathbf{B}, p)$ satisfies the conditions in $\mathcal{X}$. Hence $\mathbf{B}(p) \in \mathcal{K}_{2}$, and clearly $|B(p)|=k$.

Lemma 2.3. Every member of $\mathcal{K}_{1}$ is finite and simple.

Proof. Let $\mathbf{B}(p) \in \mathcal{K}_{1}$. If $\left|B_{p}\right|=1$ then certainly $\mathbf{B}(p)$ is finite and simple. Suppose $\left|B_{p}\right|>1$. Then by Lemma $2.1, \hat{1} \in B_{p}$ and there exist a unique $n \geq 3$ and a unique $(n+1)$-tuple $\bar{f}$ in $B(U)$ such that $\Phi_{n}(\bar{f})$. Lemma 2.1 and its proof also imply that if $T$ is a fundamental operation of $\mathbf{B}$ and $\bar{g}$ is a tuple from $B(U)$ of the appropriate arity, then $T(\bar{g}) \in B(U)$ only if $T=F_{n}$ and $\bar{g}=\bar{f}$, or $T=G_{n, i}$ for some $i \leq n$ and $\bar{g}=\langle\bar{f}, \hat{1}\rangle$. Thus $B_{p} \subseteq\left\{f_{0}, \ldots, f_{n}, \hat{1}\right\}$, so $\mathbf{B}(p)$ is finite. Since each $f_{i} \gg \hat{1}$ because of $F_{n}$, and $\hat{1} \gg f_{i}$ because of $G_{n, i}$, and since $\hat{1} \in B_{p}$, it follows that $\gg$ is symmetric on $B_{p}$; hence $\mathbf{B}(p)$ is simple.

Combining Lemmas 2.2 and 2.3 with Theorem 1.1 yields:

Theorem 2.4. Let $\mathbf{A}_{1}^{+}$be any finite algebra witnessing Theorem 1.1 for the $M$ algebra $\mathbf{A}$ and set of conditions $\mathcal{X}$ defined in this section. Then:

1. $\mathrm{V}\left(\mathbf{A}_{1}^{+}\right)$contains a $k$-element simple algebra for every $k \geq 6$, but contains no infinite simple algebras.

2. Every subdirectly irreducible member of $\mathrm{V}\left(\mathbf{A}_{1}^{+}\right) \backslash \mathrm{HS}\left(\mathbf{A}_{1}^{+}\right)$is simple.

Actually, more is true. The operations $P, Q$ (which until now have played no useful role) guarantee the following:

Proposition 2.5. If $\mathbf{A}_{1}^{+}$is the particular 7-element algebra witnessing Theorem 1.1 constructed in [5] (with $\mathbf{A}$ and $\mathcal{X}$ as defined in this section), then every subalgebra of $\mathbf{A}_{1}^{+}$is simple. Hence $\mathrm{V}\left(\mathbf{A}_{1}^{+}\right)$is semisimple, has simple members of every finite cardinality greater than 5, but has no infinite simple members.

Proof. Let $\mathbf{A}_{1}^{+}$be the algebra witnessing Theorem 1.1 constructed in [5]. It suffices to know the following about $\mathbf{A}_{1}^{+}$: the universe of $\mathbf{A}_{1}^{+}$is $A_{1}^{+}=\{0,1, a, b, \tilde{1}, \tilde{a}, \tilde{b}\}$, the type of $\mathbf{A}_{1}^{+}$includes $\wedge, P, Q, F_{3}$ and $G_{3,0}$, and

1. $\left\langle A_{1}^{+}, \wedge\right\rangle$ is a height-1 meet semilattice with least element 0 .

2. The operations $P, Q, F_{3}$ and $G_{3,0}$ of $\mathbf{A}_{1}^{+}$agree on $\{0,1, a, b\}$ with the corresponding operations of $\mathbf{A}$.

3. The type of $\mathbf{A}_{1}^{+}$also includes the operations $\mathrm{J}$ (ternary) and $\nu$ (unary), defined as follows:

$$
\begin{aligned}
\mathrm{J}(x, y, z) & = \begin{cases}x & \text { if } x=y \text { or }(x=z \text { and }\{x, y\}=\{r, \tilde{r}\} \\
& \text { for some } r \in\{1, a, b\}), \\
0 & \text { otherwise, }\end{cases} \\
\nu(x) & = \begin{cases}r & \text { if } x=\tilde{r} \text { with } r \in\{1, a, b\}, \\
x & \text { otherwise. }\end{cases}
\end{aligned}
$$

Now suppose that $\mathbf{B} \leq \mathbf{A}_{1}^{+}$and $|B|>1$. Then $\langle B, \wedge\rangle$ is also a height-1 meet semilattice with least element 0 . Thus if $\theta \in$ Con $\mathbf{B}$ and $X=0 / \theta$, then $\theta=0_{B} \cup X^{2}$. So to show that $\mathbf{B}$ is simple it suffices to show that $X \neq\{0\}$ implies $X=B$. 
Consider the following unary polynomials of $\mathbf{A}_{1}^{+}: \lambda_{1}(x)=F_{3}(x, b, b, b), \lambda_{2}(x)=$ $G_{3,0}(b, b, b, b, x), \lambda_{3}(x)=P(1, x), \lambda_{4}(x)=Q(x, a), \lambda_{5}(x)=\nu(x)$, and for each $r \in\{1, a, b\}, \mu_{r}(x)=\mathrm{J}(\tilde{r}, x, \tilde{r})$. These polynomials satisfy (i) $\lambda_{i}(0)=\mu_{r}(0)=0$ for all $i$ and $r$, and (ii) $\lambda_{1}(b)=1, \lambda_{2}(1)=b, \lambda_{3}(a)=1, \lambda_{4}(1)=a$, and $\lambda_{5}(\tilde{r})=r$ and $\mu_{r}(r)=\tilde{r}$ for each $r \in\{1, a, b\}$.

These facts imply: (i) if $\{1, b\} \subseteq B$, then $1 \in X$ if and only if $b \in X$; (ii) if $\{1, a\} \subseteq B$, then $1 \in X$ if and only if $a \in X$; (iii) if $\{r, \tilde{r}\} \subseteq B(r \in\{1, a, b\})$, then $r \in X$ if and only if $\tilde{r} \in X$. Finally, note that (iv) $\tilde{r} \in B$ implies $r \in B$ for any $r \in\{1, a, b\}$ (because of $\nu$ ), and (v) $b \in B$ implies $1 \in B$ (because $F_{3}(b, b, b, b)=1$ ). This information is sufficient to conclude that $X \neq\{0\}$ implies $X=B$.

\section{Nonsemisimple VARIETy OF FINITE TyPe}

The example given in this section is a variation of McKenzie's 8-element algebra described in $[2, \S 6]$. Let $\mathbf{A}$ be the M-algebra with universe $A=\{0,1,2, C, D, H, K\}$ and operations $\wedge, 0, \cdot$ (binary) and $t$ (ternary) where $\cdot$ and $t$ are defined as follows:

$$
\begin{aligned}
& 1 \cdot C=K \cdot D=C, 2 \cdot D=H \cdot C=D, \text { and } x \cdot y=0 \text { otherwise. } \\
& t(1, C, C)=1, t(2, D, D)=2, t(H, C, D)=H, t(K, D, C)=K \text {, and } \\
& \quad t(x, y, z)=0 \text { otherwise. }
\end{aligned}
$$

Let $U=A \backslash\{0\}$. Note that if $\mathbf{B}$ is a subpower of $\mathbf{A}$ and $f, g, h \in B(U)$, then $t(f, g, h) \in B(U)$ if and only if $f \cdot g=h$, in which case $t(f, g, h)=f$. Thus for any $p \in B(U)$, the operation $t$ is defined in $\mathbf{B}(p)$ by the rule

$$
t(x, y, z)= \begin{cases}x & \text { if } x \cdot y=z \neq 0 \\ 0 & \text { otherwise }\end{cases}
$$

For each $n<\omega$ let $\mathbf{S}_{n}$ be the M-algebra of type $\{\wedge, 0, \cdot, t\}$ having universe $\{0\} \cup\left\{a_{i}: i<n\right\} \cup\left\{b_{i}: i \leq n\right\}$, with . defined by $a_{i} \cdot b_{i+1}=b_{i}$ for $i<n$ and $x \cdot y=0$ otherwise, and with $t$ defined by $(*)$. The nonzero elements of $\mathbf{S}_{n}$ are pictured in Figure 1 (where an arrow $c \rightarrow d$ indicates $x \cdot c=d$ for some $x$, while a double-headed arrow $c \rightarrow d$ indicates $c \cdot x=d$ for some $x$ ). Also let $\mathbf{S}_{\omega}=\bigcup_{n<\omega} \mathbf{S}_{n}$.

For $0<n<\omega$ let $\mathbf{C}_{n}$ be the M-algebra of type $\{\wedge, 0, \cdot, t\}$ having universe $S_{n} \cup\left\{a_{n}\right\}$, with $\cdot$ defined by $a_{i} \cdot b_{i+1}=b_{i}$ for $i<n$ and $a_{n} \cdot b_{0}=b_{n}$ and $x \cdot y=0$ otherwise, and with $t$ defined by $(*)$. The corresponding picture of the nonzero elements of $\mathbf{C}_{n}$ would consist of Figure 1 plus the point $a_{n}$ placed directly beneath $b_{n}$, a double-headed arrow $a_{n} \rightarrow b_{n}$, and an ordinary arrow $b_{n} \longleftarrow b_{0}$ 'cycling back' from $b_{0}$ to $b_{n}$.

Lemma 3.1. 1. $\mathbf{S}_{0}$ and each $\mathbf{C}_{n}(0<n<\omega)$ are simple.

2. $\mathbf{S}_{n}$ is subdirectly irreducible but not simple for $1 \leq n \leq \omega$.

Proof. Clearly $\mathbf{S}_{0}$ is simple. Let $\mathbf{B}$ be one of $\mathbf{S}_{n}(1 \leq n \leq \omega)$ or $\mathbf{C}_{n}(1 \leq n<\omega)$. B is an M-algebra and is trivially a subpower of itself; hence the relations $\gg$ and $\ggg$ are defined on $B \backslash\{0\}$ in the usual way. Furthermore, (i) $\mathbf{B}$ is subdirectly irreducible

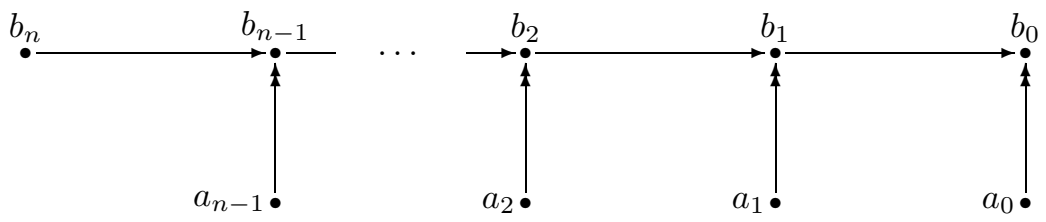

FiguRE 1. The nonzero elements of $\mathbf{S}_{n}$ 
if and only if there exists $y \in B \backslash\{0\}$ such that $x \ggg y$ for all $x \in B \backslash\{0\}$, and (ii) $\mathbf{B}$ is simple if and only if $\mathbf{B}$ is subdirectly irreducible and $\ggg$ is symmetric.

It will be useful to view Figure 1. If $x, y \in B \backslash\{0\}$ and either $x \rightarrow y$ or $x \rightarrow y$, then $x \gg y$ by definition. Thus $x \ggg b_{0}$ for all $x \in B \backslash\{0\}$, so $\mathbf{B}$ is subdirectly irreducible. In fact, the relation $\gg$ consists precisely of the $\rightarrow$ - and $\rightarrow$-edges pictured in Figure 1, reflexive loops $x \gg x$ arising from $x \wedge x=x$, and lastly the edges $b_{i+1} \gg a_{i}$ and $b_{i} \gg a_{i}$ from $t\left(a_{i}, b_{i+1}, b_{i}\right)=a_{i}$. It should now be clear that $\ggg$ is symmetric on $\mathbf{C}_{n}$ but not on $\mathbf{S}_{n}$.

Now let

$$
\mathcal{X}=\left\{\mathcal{B}_{x \in\{1,2\}}, \mathcal{C}_{x=z \& y=w}^{x \cdot y=z \cdot w}, \mathcal{C}_{y=v \& z=w}^{t(x, y, z)=t(x, v, w)}\right\},
$$

let $\mathcal{K}_{1}$ be the class of all algebras $\mathbf{B}(p)$ where $\mathbf{B} \leq \mathbf{A}^{I}, p \in B(U)$, and $(\mathbf{B}, p)$ satisfies the conditions in $\mathcal{X}$, and let $\mathcal{K}_{2}=\left\{\mathbf{B}(p) \in \mathcal{K}_{1}: B_{p}=B(U)\right\}$.

Lemma 3.2. Both $\mathcal{K}_{1}$ and $\mathcal{K}_{2}$ consist, up to isomorphism, of the algebras $\mathbf{S}_{n}$ ( $n \leq$ $\omega)$ and $\mathbf{C}_{n}(1 \leq n<\omega)$.

Proof. Suppose first that $n \leq \omega$. Let $N=\min (n+1, \omega)$ and define $a_{i} \in A^{N}(i<n)$ and $b_{i} \in A^{N}(i<N)$ by

$$
\begin{aligned}
& a_{i}(j)=\left\{\begin{aligned}
1 & \text { if } j<i, \\
H & \text { if } j=i, \\
2 & \text { if } i<j<N,
\end{aligned}\right. \\
& b_{i}(j)=\left\{\begin{aligned}
C & \text { if } j<i, \\
D & \text { if } i \leq j<N .
\end{aligned}\right.
\end{aligned}
$$

Let $V=\left\{a_{i}: i<n\right\} \cup\left\{b_{i}: i<N\right\}, B=V \cup\left(A^{N} \backslash U^{N}\right)$, and $p=b_{0}$. Then $\mathbf{B} \leq \mathbf{A}^{N}, B(U)=B_{p}=V, \mathbf{B}(p) \cong \mathbf{S}_{n}$, and $(\mathbf{B}, p)$ satisfies the conditions in $\mathcal{X}$. Thus $\mathbf{S}_{n}$ is isomorphic to a member of both $\mathcal{K}_{1}$ and $\mathcal{K}_{2}$.

The argument for $\mathbf{C}_{n}(1 \leq n<\omega)$ is similar but with the elements $a_{i}, b_{i} \in A^{n+1}$ $(i \leq n)$ defined by

$$
\begin{aligned}
& a_{i}(j)=\left\{\begin{aligned}
K & \text { if } j=i, \\
H & \text { if } j \equiv i+1(\bmod n+1), \\
2 & \text { otherwise }
\end{aligned}\right. \\
& b_{i}(j)= \begin{cases}C & \text { if } j=i, \\
D & \text { otherwise }\end{cases}
\end{aligned}
$$

Conversely, suppose $\mathbf{B} \leq \mathbf{A}^{I}$ and $p \in B(U)$ are such that $(\mathbf{B}, p)$ satisfies the conditions in $\mathcal{X}$. If $\left|B_{p}\right|=1$ then $\mathbf{B}(p) \cong \mathbf{S}_{0}$. For the remainder of this proof assume that $\left|B_{p}\right| \geq 2$. Let $X=\{C, D\}^{I} \cap B_{p}$ and $Y=\{1,2, H, K\}^{I} \cap B_{p}$, and note that if $f, g \in B_{p}$ and $f \neq g$ and $f \gg g$, then $\{f, g\} \subseteq X \cup Y$. Thus $B_{p}=X \cup Y$; moreover, both $X$ and $Y$ are nonempty, and the nonreflexive $\gg$-edges in $B_{p}$ consist precisely of the $\rightarrow$-edges between points in $X$, the $\rightarrow$-edges from points in $Y$ to points in $X$, and the remaining $\gg$-edges (corresponding to the operation $t$ ) from points in $X$ to points in $Y$.

We shall first examine the $\rightarrow$-edges.

Claim 1 . There is no $g \in X$ satisfying $g \rightarrow g$.

Otherwise, $f \cdot g=g$ for some $f \in Y$. This can only happen if range $(f) \subseteq\{1,2\}$. But the existence of $f$ with this property is prohibited by $\mathcal{B}_{x \in\{1,2\}}$. 
Claim 2. For each edge $g \rightarrow h$ in $X$ there exists a unique $f \in Y$ satisfying $f \cdot g=h$.

Existence follows from the definition of $\rightarrow$. Suppose $f \cdot g=h=f^{\prime} \cdot g$. Then $\mathcal{C}_{x=z \& y=w}^{x \cdot y=z \cdot w}$ and the fact that $f, f^{\prime}, g, h \in B_{p}$ imply $f=f^{\prime}$.

Claim 3. For each $f \in Y$ there exists a unique edge $g \rightarrow h$ in $X$ such that $f \cdot g=h$.

If there were no such edge $g \rightarrow h$, then $f$ would be disconnected from $B_{p} \backslash\{f\}$ with respect to $\gg$, which is impossible. This proves existence; uniqueness is established using $\mathcal{C}_{y=v \& z=w}^{t(x, y, z)=t(x, v, w)}$.

At this point it is obvious that (i) there is a one-to-one correspondence between the $\rightarrow$-edges in $X$ and the points in $Y$; (ii) the nonreflexive $\gg$-edges of $B_{p}$ are the $\rightarrow$-edges in $X$ plus, for each such edge $g \rightarrow h$, the edges $f \rightarrow h, g \gg f$ and $h \gg f$, where $f$ is the unique point in $X$ corresponding to $g \rightarrow h$; (iii) we may assume with no loss of generality that $p \in X$; (iv) for each $f \in X \backslash\{p\}$ there is a directed $\rightarrow$-path from $f$ to $p$.

It remains only to show that $\langle X, \rightarrow\rangle$ is either a finite chain, an infinite chain with end point but no initial point, or a cycle. By observation (iv) above, it suffices to show that (v) $g \rightarrow h$ and $g^{\prime} \rightarrow h$ imply $g=g^{\prime}$; and this is guaranteed by $\mathcal{C}_{x=z \& y=w}^{x \cdot y=z \cdot w}$.

Combining Lemmas 3.1 and 3.2 with Theorem 1.1 gives the desired example.

Theorem 3.3. Let $\mathbf{A}_{1}^{+}$be any finite algebra witnessing Theorem 1.1 for the $M$ algebra $\mathbf{A}$ and set of conditions $\mathcal{X}$ defined in this section. Then:

1. $\mathrm{V}\left(\mathbf{A}_{1}^{+}\right)$contains a finite simple algebra of every odd cardinality greater than 3, but contains no infinite simple algebras.

2. The type of $\mathbf{A}_{1}^{+}$is finite.

If $\mathbf{A}_{1}^{+}$is the particular algebra witnessing Theorem 1.1 constructed in [5], then $\mathbf{A}_{1}^{+}$has 13 elements and 11 fundamental operations, of arities 5, 5, 4, 4, 3, 3, 3, 2, $2,1,0$ respectively.

\section{REFERENCES}

[1] D. Hobby and R. McKenzie, The Structure of Finite Algebras, Contemp. Math., vol. 76, Amer. Math. Soc. (Providence, R.I.), 1988. MR 89m:08001

[2] R. McKenzie, The residual bounds of finite algebras, Int. J. Algebra and Computation 6 (1996), 1-28. MR 97e:08002a

[3] R. McKenzie, G. McNulty and W. Taylor, Algebras, Lattices, Varieties. Vol. I, Wadsworth \& Brooks/Cole (Monterey, CA), 1987. MR 88e:08001

[4] M. Valeriote, A residually small, finitely generated, semi-simple variety which is not residually finite, Int. J. Algebra and Computation 6 (1996), 563-569. MR 97h:08005

[5] R. Willard, On McKenzie's method, Periodica Math. Hungarica 32 (1996), 149-165. CMP 97:01

Department of Pure Mathematics, University of Waterloo, Waterloo, Ontario, CANADA N2L 3G1

E-mail address: rdwillar@gillian.math.uwaterloo.ca 